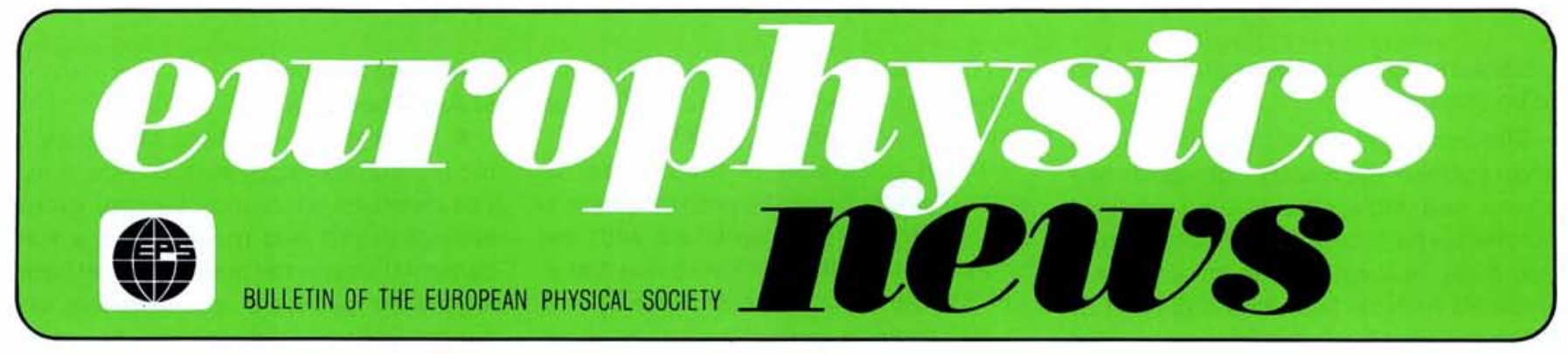

\title{
Surface Physics at BESSY
}

\section{W. Braun and A.M. Bradshaw, Berlin West}

\author{
(Berliner Elektronenspeicherring-Gesellschaft für Synchrotronstrahlung m.b.H.)
}

The physics and chemistry of surfaces has become a field of major significance during the past decade. Surfaces and interfaces have important implications for many modern technologies from electronic engineering, where semiconductor physicists move constantly to smaller devices, through industrial chemistry, where surface chemists seek an improved understanding of catalysis, to materials science, where, for example, a more thorough knowledge of the mechanism of corrosive oxidation is required. The techniques of surface treatment and coatings as well as an understanding of thin-film adhesion are vitally important for the further development of many other technologies. At the same time there exist a large number of challenging problems in basic research on surfaces. This list helps to explain the large number of physicists and chemists presently working in this very topical field. A powerful tool in the hands of the researchers is the application of surface

\section{Conferences}

Fees for IOMs

Although traditionally June has been a Meetings Issue, as previously announced in Europhysics News it is now a "Green" Issue and includes only paying conference announcements:

(Sw.Fr. 100.--insertion).

Organizers of EPS Conferences are reminded that following the decision of Council in March, registration fees for Individual Ordinary Members of EPS must be lower than the general fee by a figure close to the yearly subscription. sensitive spectroscopies which exploit the photon-stimulated excitation of energy levels in matter using synchrotron radiation ${ }^{1}$ ).

The earliest applications of synchrotron radiation in the ultraviolet and soft $\mathrm{X}$-ray regions were in optical spectroscopy, where it was used in reflection studies of solids as well as in absorption spectroscopy of atoms and molecules. Absorbed photons, however, can also cause electrons and ions to be emitted, and this forms the basis for the surfacesensitive photon-stimulated emission spectroscopies germane to this article. In particular, photon-stimulated electron emission ("photoemission") has proved to be a versatile and powerful probe of the electronic structure of solids as well as of the electronic and geometric structure of surfaces and adsorbed layers.

Photoemission (or photoelectron spectroscopy) directly probes the energy levels and symmetry of electron wavefunctions in matter. Of particular interest are the outermost valence electrons because they determine chemical and physical properties such as electrical conductivity, magnetism, optical properties and chemical reaction mechanisms at the surface and in the bulk. Photoelectron spectroscopy determines the so-called electron binding energy (i.e. the energy difference between the electronic ground state and the excited hole state of the system) which, in a first (and relatively good) approximation known as Koopmans' theorem, may be equated with the energies of the oneelectron levels of the ground state. In the case of a crystalline solid these valence energy levels form bands characterised by their energy versus momentum dis- persions, $E(\mathbf{k})$. Information as to the symmetry of the level(s) may be obtained if a source of polarised light (as with synchrotron radiation) is available. In addition, photoelectron spectroscopy applied to core levels allows the inner shell binding energies to be precisely determined. These vary according to the valence field at the site of the ionised core level and give rise to "chemical shifts". Such shifts are invaluable in surface analysis as well as in phenomenological studies of bonding mechanisms in molecules, in solids and at surfaces. The mean free path of photoelectrons in matter and thus the surface sensitivity of photoemission is strongly dependent upon the kinetic energy of the excited electron and shows a minimum some 10-20 eV above the excitation threshold. Synchrotron radiation enables us to tune into this minimum in order to vary the effective sampling depth down to about one atomic layer. It also allows various effects associated with many body ex-

\begin{tabular}{|lr|}
\hline Contents & \\
Surface Physics at BESSY & 1 \\
Directory & 5 \\
Officers and Member & 5 \\
Organizations & 6 \\
Advisory Committees & 7 \\
Division and Section Boards & 9 \\
Associate Members & 10 \\
Europhysics Journals & 10 \\
Membership Terms and & 11 \\
Advertising & 12 \\
Conference Up-date & \\
Teaching Abroad & 13 \\
Vortices in Rotating & \\
Superfluid ${ }^{3} \mathrm{He}$ & \\
\hline
\end{tabular}


citations accompanying photoionisation to be investigated.

The determination of the bond lengths and surface geometry of adsorbed atoms and molecules is an important problem in surface science ${ }^{2}$ ). The extended $\mathrm{X}$-ray absorption fine structure associated with surface atoms (SEXAFS) helps to solve such questions provided that surface sensitive detection techniques are used. The method relies on the oscillations in the X-ray absorption coefficient on the high energy side of an absorption edge that arise from the scattering of the excited electrons by neighbouring atoms. The structure may extend up to $1000 \mathrm{eV}$ above the edge with an amplitude of about $10 \%$ of the total absorption. SEXAFS enables coordination numbers as well as bond lengths to be determined to an accuracy of \pm 0.03 $\AA$. It can also provide information on the immediate environment of an individual atomic species in a multicomponent system. For such experiments, the tunability of the light source is clearly essential.

The impact of ionising radiation on adsorption layers, consisting of atoms or molecules on metal and semiconductor surfaces, also causes desorption of charged particles and neutrals ${ }^{3}$ ). This process is well known as a degassing effect in electron or positron accelerators. Photon and electron stimulated desorption can also be used as a probe for adsorbate states. Photon-stimulated desorption with a high intensity tunable light source such as a storage ring has considerable advantages over electroninduced desorption, because of the sharp onset of the structure observed in the spectra. Unfortunately, the crosssections for this process are about four orders of magnitude lower than the corresponding total photoionisation crosssections. The high intensity and high brightness tunable light sources required for such studies are at present only available in the form of dedicated electron storage rings.

$B E S S Y$, an $800 \mathrm{MeV}$ electron storage ring in Berlin, is one such source and is now in its second year of user operation. It has been built as a national facility developed for experiments in basic research, X-ray lithography and radiometry. In the basic research area, there are 16 experimental stations now in operation for experiments in X-ray microscopy, solid state physics and surface science as well as in atomic and molecular spectroscopy. The following properties make synchrotron radiation a unique source for spectroscopies at photon energies above $\hbar \omega=5 \mathrm{eV}$ :
- Continuous spectrum from the infrared to the $\mathrm{X}$-ray region (at BESSY up to photon energies of $\sim 2000 \mathrm{eV}$ ).

- Good collimation of the emitted radiation tangential to the path of the emitting electron. This, combined with the source size is called the emittance and at BESSY, the horizontal emittance $\varepsilon_{x}=4$ $\times 10^{-8} \pi$ rad $\mathrm{m}$ gives rise to a very high brightness of around $5 \times 10^{12}$ photon $/ \mathrm{s}$ per ( $\%$ bandwidth, $\mathrm{mm}^{2}, \mathrm{~m} \mathrm{rad}^{2}$ ).

- Linear polarisation in the orbit plane, elliptical polarisation above and below the orbit plane.

- Pulsed time structure (at BESSY pulse lengths of about 100 ps at repetition rates of $2 \mathrm{~ns}$ in multi-bunch operation, and $208 \mathrm{~ns}$ in single bunch operation).

- Absolute calculability of all properties of the source. (At BESSY this feature is utilised by the Physikalisch-Technische Bundesanstalt (the German National Bureau of Standards) which plans to establish synchrotron radiation as an intensity standard in the VUV).

Most experiments require monochromatic radiation. In the spectral range up to $\hbar \omega \sim 1000 \mathrm{eV}$, grating monochromators are used to disperse the emitted white radiation. In this region one can distinguish roughly between "normalincidence" (5 eV $\leq \hbar \omega \leq 50 \mathrm{eV})$ and "grazing-incidence" monochromators $\left.(20 \mathrm{eV} \leq \hbar \omega \leq 800 \mathrm{eV})^{4}\right)$. Above $\hbar \omega=$ $20 \mathrm{eV}$, the reflectivity of optical components at near normal incidence is very low so that in order to optimise reflectivity at higher photon energies, the angle of incidence has to be increased (to an extent dependent on the required energy range) thus sacrificing simplicity and resolving power through aberrations in the optical system. In the "grazing-incidence" region, complicated geometrical surfaces are necessary for the optical components which are consequently difficult to manufacture with the required low degree of surface roughness. Thus the resolving power $(=\lambda / \Delta \lambda)$ here is presently limited to less than $10^{3}$ whereas in the "normal-incidence" region, resolving powers better than $10^{4}$ can be obtained.

With monochromatised synchrotron radiation, a large variety of experiments are carried out in several centres in Europe, the US and Japan in such different areas as chemistry, physics, biology and medicine. Many of them cannot be performed using present conventional laboratory sources. In this article we shall concentrate on activities in the field of surface science (but include some work in solid state physics) at the synchrotron radiation source BESSY.

\section{Electronic Structure of Adsorbed Layers}

A major goal of surface chemistry is the understanding of surface reactivity: The chemical adsorption (or chemisorption) of atoms and molecules is a fundamental step in the mechanism of heterogeneous catalysis and corrosive oxidation. Prior to such studies, the clean surface of the metal or semiconductor (usually in the form of a single crystal) has to be well characterised. Photoelectron spectroscopy has become a major probe in the study of the electronic structure of such adsorption systems. If the momentum (or wave-vector $\mathbf{k}$ ) of the emitted photoelectron is also measured, then besides probing the energy levels of the system, the orientation of the adsorbed molecule may be determined via dipole selection rules. If the adsorbate forms a two-dimensional ordered array at high coverages, then it is also possible with angle-resolved techniques to measure two-dimensional energy band dispersions. The main advantages of synchrotron radiation here are its wide spectral range and its high degree of polarisation. It is thus possible to optimise surface sensitivity or to tune into either the maximum in the photoionisation cross-section of the adsorbate level or the minimum in that of the substrate levels.

At BESSY the adsorption of chlorine on a copper single crystal of (100) surface orientation has recently been studied by Baalmann et al. The chlorine atoms form an ordered overlayer at a coverage of 0.5 relative to the outermost layer of copper atoms. The adlayer consists of a square array rotated by $45^{\circ}$ relative to that of the substrate and is

Fig. 1 - Photoelectron spectra obtained from a clean and chlorine covered $\mathrm{Cu}(100)$ surface (A. Baalmann et al., to be published).

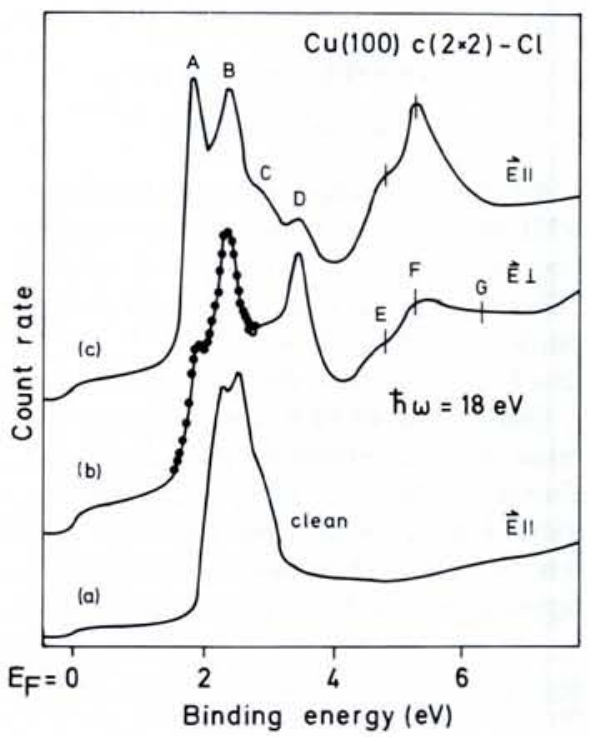


denoted as c $(2 \times 2) \mathrm{Cl} / \mathrm{Cu}(100)$. Such a structure is the first step in the epitaxial growth of copper chloride.

Angle-resolved photoelectron spectra, where the take-off angle was normal to the surface, were taken for two different incident light geometries (polarization vector parallel $\left(E_{1}\right)$ or nearly nor$\mathrm{mal}\left(\mathrm{E}_{\perp}\right)$ to the surface) and a variety of photon energies. Fig. 1 shows as an example the electron energy distribution curves (EDCs) at $\hbar \omega=18 \mathrm{eV}$. Spectrum (a) represents the clean copper surface with strong emission from the $\mathrm{d}$ bands between 1.8 and $2.5 \mathrm{eV}$ superimposed on the unstructured less intense emission from the sp band. The chlorinederived features $A, D, E, F, G$ are due to the $\mathrm{Cl} 3 p$ orbitals which have been shifted, broadened and split due to the interaction with the substrate. Using dipole selection rules the symmetry behaviour of the chlorine-derived peaks labelled $A, F$ and $D, G$ in Fig. 1 can be identified. The peaks $F$ and $A$ correspond to the bonding and anti-bonding combinations, respectively, of the $\mathrm{Cl} 3 \mathrm{p}_{\mathrm{x}}, \mathrm{p}_{\mathrm{y}}$ orbitals with substrate states.

The additional features in the spectra (b) and (c) also show dispersion effects. $A$ high coverage ordered overlayer such as c $(2 \times 2) \mathrm{Cl} / \mathrm{Cu}(100)$ implies overlapping orbitals on adjacent adspecies and thus the formation of two-dimensional Bloch states. The latter are characterised by a two-dimensional wave vector, $\mathbf{k}_{\|}$, in the surface Brillouin zone of the adlayer. In photoemission from an ordered surface, it is generally assumed that the parallel component of momentum is conserved and since $\mathbf{k}_{\|}$can be determined experimentally, it is clear that the dispersion of the bands in the different symmetry directions can be determined directly from measurements at emission angles off normal. Such experiments have already been performed with laboratory line sources and give information on the extent of lateral interactions in the adlayer. The normal emission geometry of Fig. 1 corresponds to a probing of states at the centre of the surface Brillouin zone $\left(\mathbf{k}_{\|}=0\right)$.

Another interesting experiment using a two-dimensional display analyser has been performed by Rieger et al. to determine the orientation of carbon monoxide adsorbed on different single crystal faces of platinum. In this experiment, the photon energy is tuned into the maximum of the photoionisation crosssection of a particular adsorbate level and photoelectrons at a particular energy are collected within an emission cone of $88^{\circ}$. By subtracting the contributions from the clean surface, the angular dis-

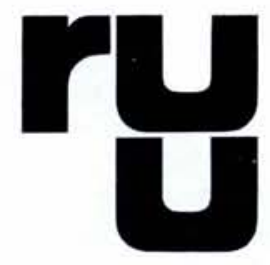

Rijksuniversiteit Utrecht

The Department of Physics and Astronomy of the Rijksuniversiteit Utrecht invites applications for the position of

\title{
PROFESSOR
}

\section{OF THEORETICAL PHYSICS}

\section{in the Institute for Theoretical Physics, starting September 1984.}

The successful applicant will conduct and stimulate research in the area of statistical mechanics and condensed matter, and participate in teaching general and theoretical physics courses, mainly at the graduate level. $\mathrm{He} / \mathrm{she}$ is expected to take part in the policy-making and administrative duties of the Institute and the Department.

Candidates should have an international reputation in the area of statistical physics and condensed matter, with a broad interest in theoretical physics. They should be willing to learn Dutch within two years, so as to be able to teach effectively in this language.

Further details can be obtained from the chairman of the selection committee :

\author{
Professor M.H. Ernst, \\ Institute for Theoretical Physics, \\ Princetonplein 5, \\ NL-3584 CC Utrecht \\ Tel. : (30) 532284
}

Salary depending on age and experience to a maximum of Dfl. 9005.-/month (12 months).

Applications, including a curriculum vitae and a list of publications, should be sent to the chairman of the selection committee,

Professor M.H. Ernst, Institute for Theoretical Physics,

Princetonplein 5, 3584 CC Utrecht, The Netherlands, within four weeks of the publication of this announcement.

Those wishing to recommend suitable candidates are urged to contact the chairman of the selection committee. 


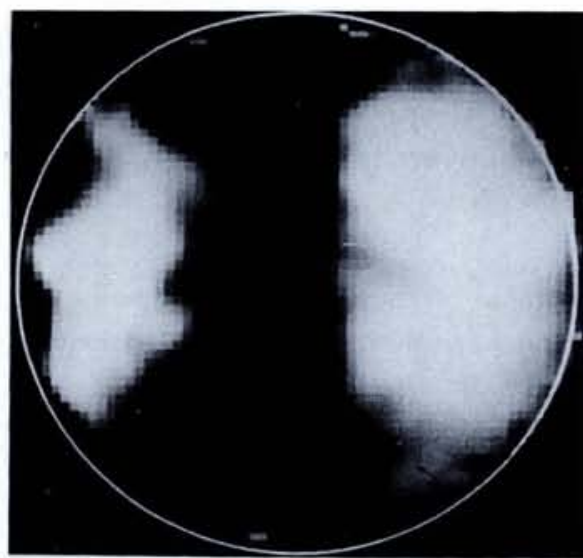

a

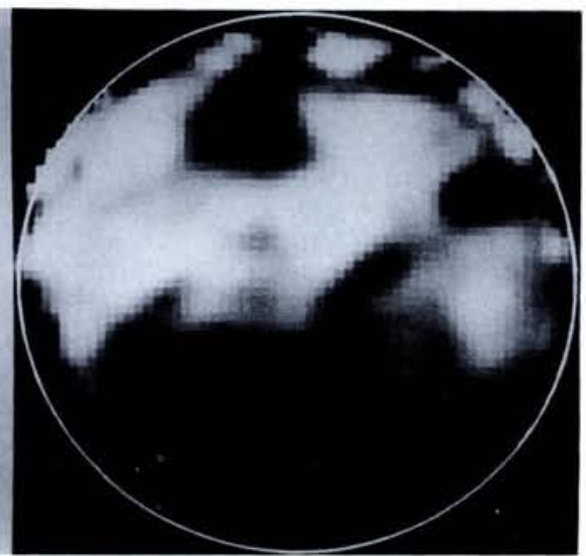

b

Fig. 2 - Angular distribution pattern of photoemitted electrons of a particular valence level of carbon monoxide (5\%) adsorbed on different low index surfaces of platinum CO/Pt (111) (a) and CO/Pt (110) (b) (D. Rieger et al., Ann. Israel Phys. Soc. 6 (1983) 182).

tributions of the photoelectrons emitted from particular molecular orbitals can be displayed, as shown in Fig. 2. By exploiting this technique in conjunction with dipole selection rules, it was found, that $\mathrm{CO}$ adsorbs upright with the $\mathrm{C}$ atom facing the (111) surface of platinum, whereas it is tilted with respect to the surface normal on the corresponding (110) surface.

\section{Mapping of Energy Bands in Solids}

The electronic structure of solids and their clean surfaces is a subject of widespread interest. Photoelectron spectroscopy using synchrotron radiation is an important method for investigating oneelectron energy bands and their symmetries. Such energy-versus-momentum dispersions $E(\mathbf{k})$ are shown in Fig. 3. They have been determined by Wern et al. in an experiment on a silver single

Fig. 3 - Experimental energy-versus-momentum dispersion relations $E(\boldsymbol{k})$ of silver along the symmetry line $\Lambda(H$. Wern et al., to be published).

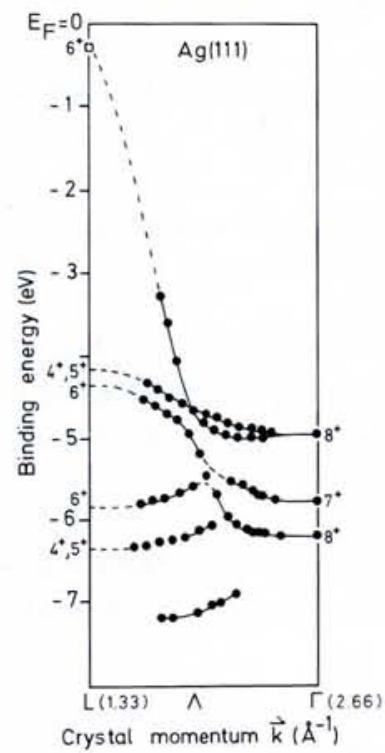

crystal with an atomically smooth (111) surface. Experimentally, such dispersion curves are determined from EDCs (e.g. spectrum (a) in Fig. 1) taken in selected emission directions at various photon energies. It is assumed that the first step in the photoemission process is bulk optical absorption involving a momentumconserving "direct" interband transition from an occupied band into an unoccupied band above the vacuum level.

The determination of the $E(\mathbf{k})$ relation for an occupied band using the photon energy dependence of EDCs is thus essentially the problem of determining the value of $\mathbf{k}$ at which the direct transition takes place, since only the parallel component of $\mathbf{k}$ is conserved in the photoemission process. For this, a knowledge of the $E(\mathbf{k})$ relation for the final state band is required. The generally strong dispersion of the unoccupied bands enables us, however, to use calculated band structures for the final state: inaccuracies of the order of $1 \mathrm{eV}$ lead to quite negligible errors in the determination of the $\mathbf{k}$ value. In a first approximation the electron-hole interaction is also neglected. In Fig. 3, the final state $E(\mathbf{k})$ has been taken from the density functional calculations of Eckardt et al. Dashed lines are used to represent the course of the bands where some uncertainty still exists or where direct transitions are not observed. The results are found to deviate by not more than 0.3-0.4 eV from those predicted by theory.

More detailed information on the band structure of both magnetic and nonmagnetic materials can be obtained when the photoelectrons are also analysed with respect to their spin. Polarised synchrotron radiation can be utilised to assign and map the bands in heavier metals which are split by spin-orbit cou- pling. Fig. 4 (top) shows the EDC for the total photoelectron current in normal emission from a $\mathrm{Pt}$ (111) surface.

\section{Resonant Photoemission}

The continuous spectrum of synchrotron radiation is essential for performing resonant photoemission experiments. In addition, its high intensity is required when such experiments are performed in the gas phase where the atomic densities are about two orders of magnitude lower than in the solid state. In these experiments the photon energy is tuned, for example, to the threshold energy for creating a core hole. In metals or adsorbed molecules on metal surfaces a core electron is then excited to a state just above the Fermi level. The resulting hole may decay via an Auger transition, thus simulating the direct emission of a valence electron. Resonant photoemission experiments have recently been performed at BESSY on europium intermetallic

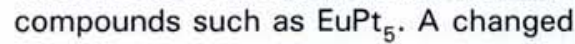
valence at the surface was observed that is caused by the strong coordination dependence of the valence on the state of Eu.

In this experiment, the resonant enhancement of the $4 \mathrm{f}$ states of Eu near the $4 d-4 f$ threshold was exploited as shown by the EDCs excited with photon energies between $120 \mathrm{eV}$ and $150 \mathrm{eV}$ in Fig. 5. The observed behaviour can be

Fig. 4-Photoelectron energy distribution curves obtained from a Pt(111) single crystal surface at $\hbar \omega=13 \mathrm{eV}$ including spin polarisation and the corresponding band structure of Pt. The dashed curve is the final state energy band shifted by $13 \mathrm{eV}$. The cross overpoints with the initial state bands indicate where the direct transitions occur in momemtum space (A. Eyers at al., Phys. Rev. Lett. 52 (1984) 1559).

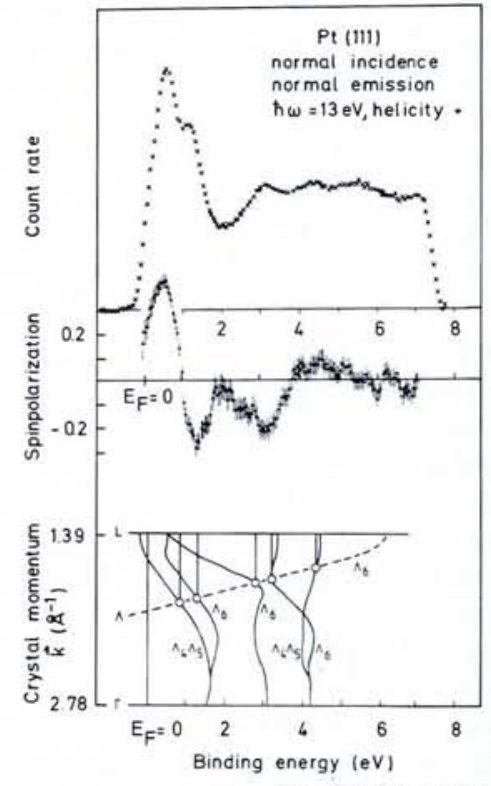

Continued on page 11 


\section{Conferences}

21-24 August 1984

Brussels, Belgium

Europhysics Conf, on Software Engineering, Methods and Tools in Computational Physics

P. van Binst, Univ. of Brussels, c.p. 230, bd. du Triomphe, B-1050 Brussels

A: 1.7 .84 / Ab: 1.3 .84 / PP: 1.8 .84 / 300 / BFr. 4000 . IOM; 7000.- others (incl. proc.)

11-14 September 1984

Aussois, France

Physics and Chemistry of High Condensed Matter

J.M. Besson, Physique des Milieux très Condensés, Tour 13 E.4, Université Paris VI,

4 place Jussieu, F-75230 Paris Cedex 05

16-20 June 1985

Joensuu, Finland

Symposium on the Foundations of Modern Physics: 50 years of the Einstein-Podolsky-Rosen Gedanken. experiment

Pekka Lahti, Department of Physical Sciences, University of Turku, SF-20500 Turku 50

17-22 June 1985

10 th Int. Workshop on Weak Interaction

M. Roos, Dept. of High Energy Physics,

Univ. of Helsinki,

Siltavuorenpenger 20 C, SF-00170 Helsinki 17 A: $1.4 .85 / \mathrm{NP} / 80 / \mathrm{Fmk} 300$.-

\section{1-6 July 1985}

Paris, France

International Conference on Nuclear Physics: Intermediate Energy Nuclear Physics with Electromagnetic Probes

A. Gerard \& C. Samour, DPhN/HE, C.E.N. Saclay, F-91191 Gif-sur-Yvette Cedex

A: 31.1.85/PP: 31.3.85/300/SFr. 210.- IOM; 230 . members 4b, 250.- others (incl. proc.); 100.- (students)

4-18 August 1985

Dronten, The Netherlands International Summerschool on Nuclear Structure on: "Frontiers in Nuclear Dynamics"

P.K.A. de Witt Huberts, NIKHEF-K, P.O. Box 4395 NL-1009 AJ Amsterdam

explained as follows. At $\hbar \omega=132 \mathrm{eV}$, only the Pt $5 \mathrm{~d}$ states are observed because at this photon energy a minimum in the photoionisation cross-section of the $4 \mathrm{f}$ levels occurs. At $\hbar \omega=140$ $\mathrm{eV}$ emission due to divalent Eu (labelled Eu $4 f^{6}$ ) is resonantly enhanced. Above $\hbar \omega=142 \mathrm{eV}$, emission due to trivalent Eu (labelled Eu $4 \mathrm{f}^{5}$ ) starts to contribute to the resonant photoemission. The fact that the onset of the corresponding Auger transition occurs at different photon energies clearly demonstrates the existence of two different valence states of europium in this compound. Since Mössbauer spectroscopy has proved the trivalent character of Eu in the bulk, the experiment demonstrates a valence change of surface atoms.

\section{Photon Stimulated Desorption}

Studies of photon-stimulated ion desorption from surfaces are only possible if high intensity light sources together with high throughput monochromators are available. In addition, the development of new instrumentation for the analysis of ion energy, angular and mass distributions is required. Treichler et al. have made studies of the molecular and fragmented ion desorption of $\mathrm{CO}$ adsorbed on the hexagonal ruthenium (100) surface. Fig. 6 shows, as an example, the intensity distributions of desorbed $\mathrm{CO}^{+}$ and fragments in the photon energy region above about $530 \mathrm{eV}$, the threshold for photoionisation of the oxygen 1s level. The molecular ion $\mathrm{CO}^{+}$starts desorbing at the threshold with an oscillating intensity distribution, whereas the onset of desorption of the fragments is delayed. From this behaviour it can be concluded that the desorption of $\mathrm{CO}$ ions is concomitant with the creation of $\mathrm{O}$ 1s core holes while the creation of the fragments is due to multi-electron excitations. Further experiments on angular distributions can give information on site- and bonding geometries of the adsorbates. Photon-stimulated ion desorption is thus a unique probe for atomic and molecular adsorbates, co-adsorbed species and chemical reactions on surfaces. In particular, adsorbed hydrogen can easily be seen with photon-stimulated desorption but is difficult to observe in photoemission and impossible to detect with Auger spectroscopy.

\section{Structural Investigations}

An exact determination of bond lengths and adsorption sites of adsorbed atoms and molecules is of paramount importance in surface studies.

Bond lengths can be obtained by Fourier transforming the SEXAFS signal from the adsorbate/substrate system above an appropriate absorption edge. The amplitude of the SEXAFS signal also contains information on the effective coordination number and depends

A recent collection of papers on the state of art of Mirror, Bumpy Torus, Spheromak and Reversed-Field Pinch physics and technology, produced by Monotypia Franchi, Citta di Castello (Perugia) Italy, for the

Varenna International School of Plasma Physics on

\section{MIRROR-BASED AND FIELD-REVERSED APPROACHES TO MAGNETIC FUSION}

Proceedings of the September 1983 COURSE and WORKSHOP ( 3 volumes $\simeq 400$ pages each). Price US \$75.-for indivisible sets (for orders received by 30 June 1984: US \$55.-)

Please order from MONOTYPIA FRANCHI, viale Umbria No. 28 CITTA DI CASTELLO (Perugia) Italy.

Payment to be remitted in advance to CASSA DI RISPARMIO, Citta di Castello (Perugia) Italy for Account No.18356-77 of MONOTYPIA FRANCHI.

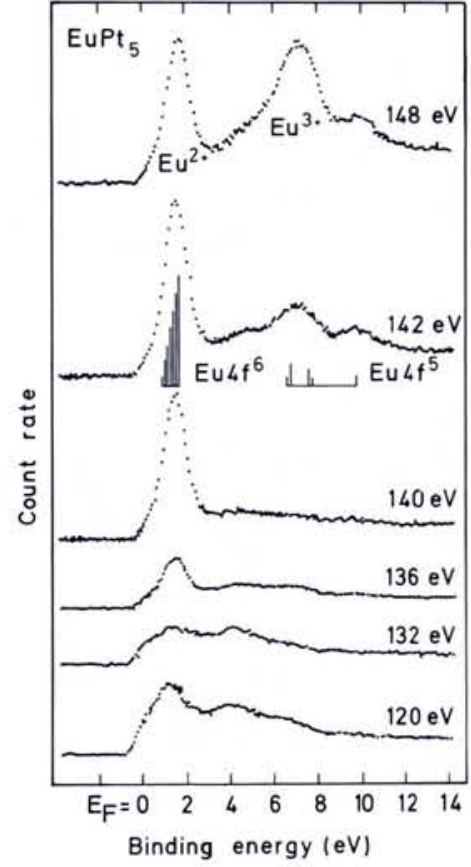

Fig. 5 - Valence-band photoelectron energy-distribution curves of EuPt $t_{5}$ excited at various photon energies between 120 and $150 \mathrm{eV}$ (W.D. Schneider et al., Phys. Rev. B 28 (1983) 2017).

critically on the direction of the electric field vector of the exciting radiation relative to the surface. This behaviour can be exploited for an unambiguous determination of the adsorption site. The SEXAFS signal is extracted from a measurement applying the surface sensitive technique of secondary electron emission with and without energy discrimination.

Fig. 6 - Intensity distributions of desorbed carbon monoxide ions and its fragments in the photon energy region above $530 \mathrm{eV}(R$. Treichler et al., to be published).

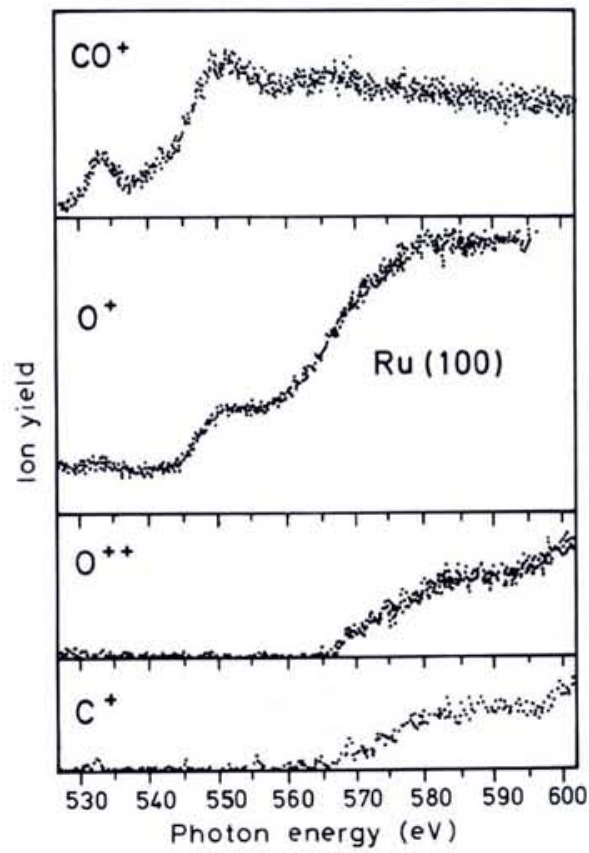


$\mathrm{Cu}(110)-0(2 \times 1)$
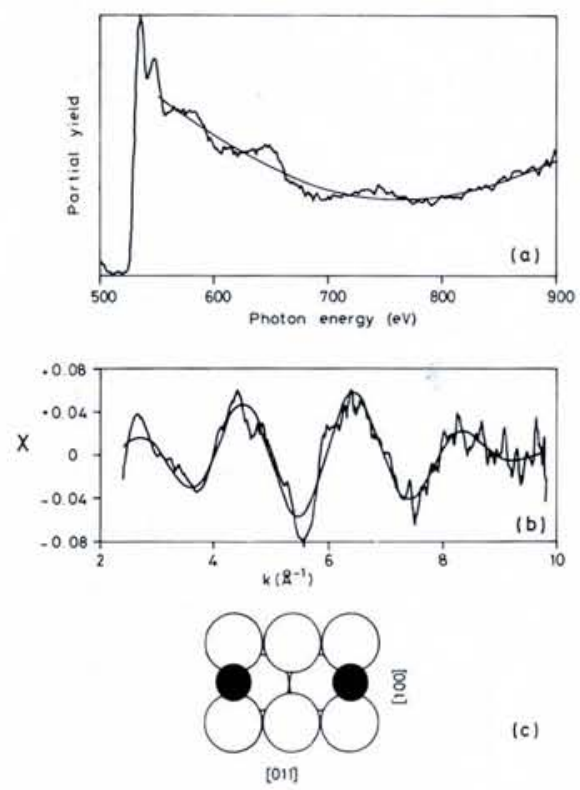

Fig. 7 - SEXAFS-signal before (a) and after (b) background subtraction of oxygen adsorbed on a $\mathrm{Cu}(110)$ single crystal together with the surface structure model (c) (U. Döbler et al., Phys. Rev. Lett. 52 (1984) 1437).

Fig. 7 shows the SEXAFS signal before (a) and after (b) background subtraction of oxygen adsorbed on a copper (110) single crystal. From these data, a bond length of $1.85 \AA$ between oxygen and copper atoms has been determined. The polarisation dependence of the SEXAFS signal indicates that the oxygen atoms sit in bridge sites along the [100] direction and are located $0.3 \AA$ above the $\mathrm{Cu}(110)$ surface (Fig. 7c).

\section{Summary}

BESSY, the German Federal Republic's national synchrotron radiation source for the VUV and soft X-ray regions, is currently in its second year of user operation. In this article we have described some of the experiments in progress and attempted to show how the properties of synchrotron radiation can be exploited in this highly topical area of research.

\section{REFERENCES}

1. Eastman D.E. and Himpsel F.J., Physics Today 641981 May.

2. Lee P.A., Citrin P.H., Eisenberger P. and Kincaid B.M., Rev. Mod. Phys. 53 (1981) 769.

3. Menzel D. and Gomer R., J. Chem. Phys. 41 (1964) 3311; Readhead P.A., Can. J. Phys. 42 (1964) 886.

4. Kunz C. (ed.), Synchrotron Radiation: Techniques and Applications (Springer Verlag, Berlin) 1979.

\title{
TEACHING ABROAD
}

\section{Franciszek Kaczmarek, Poznan}

\author{
Under the Teaching Abroad Scheme of the European Physical Society. \\ organized through the Physics Education Committee, Professor Kacz- \\ marek has spent two months at Lund University participating in the \\ faculty life there. He presents here a few of his impressions.
}

On the invitation of Professor Indrek Martinsson and Professor Hans Ryde of Lund University I spent two months (September and October 1983) in the Department of Physics of Lund University. Although the main goal of my visit was to become familiar with the teaching in Lund and to take an active part in selected teaching activities, I had also the opportunity to see the research work at the Institute of Technology and within the Atomic Spectroscopy Group where the very well equipped laboratories create a solid base for good spectroscopic investigations. It should also be noted that practical applications of modern spectroscopy methods in air pollution measurements, in treating tumours and in many other fields appeared surprisingly well developed. However, I was mainly associated with the educational group of Ingmar Johansson and I spent many hours in the student laboratories, especially during their introductory exercises and later on when they performed more advanced experiments.

As in Poland, where, for example, the number of freshmen entering the physics faculty in the University in Poznan is 40-50 a year, it would seem that physics studies are not very popular in Sweden. The period when almost everybody was excited by the spectacular achievements in physics appears to be over and the youth is more pre-occupied by the possible world disaster which - indirectly - may come from physics. Moreover, it is now very hard to find jobs for physicists in industry and research laboratories.

There are important differences between the Lund schedule and our schedule in Poznan. For example, the basic physics course in Poznan is spread over a two-year period at the rate of about four lectures plus two numerical exercises per week. In Lund, the basic course also takes two years, but $50 \%$ is mathematics. During the physics part, the number of lessons is about 16/week of which some are numerical exercises. In some weeks, the lecturing gives place to laboratory work; this occupies the equivalent of three full days in any week. The practical part of the Lund education programme consists of an Introductory
Laboratory (16 experiments performed within several days), followed by Normal Laboratory with simple and more advanced experiments, and special tasks performed in research laboratories concerned with, for example, Compton scattering, bubble chambers, Ca-spectrum, beam foil spectroscopy, optical pumping, etc. By comparison, in the Polish system we have Physics Laboratory I, first year students, four hours a week over one year - Physics Laboratory II - six hours a week over one year during the second and the third year of studies - and also special tasks performed at various research laboratories.

I did not find the Swedish students to be much different from our own students except they had a much higher proficiency in English and, to my surprise, their discipline regarding lectures was unbeliveably impressive as compared to the situation in Poland. It is rather normal that about $50 \%$ of the student body do not attend lectures at our University and furthermore, some of them do not make use of their time that is devoted to lectures.

There is another important difference between the Swedish and Polish education process. Many faculty members in our country feel that a greater than ordinary teaching activity is not properly appreciated. Promotion very rarely owes a great deal to special educational work because it mainly, or solely, depends on the research record. A very good teacher has, in practice, no chance of being promoted or even of keeping his position for a long time. However, discussion on this problem has been going on now for the past year (with no practical results as yet). Nevertheless, this is not the main reason why some faculty members do not pay enough attention to teaching.

In contrast, I have found in Lund a large group of people approaching their teaching work with extraordinary involvement, very sensitive to the students' problems and trying to make the eduction as rewarding as possible. Consequently, being in Lund I had the occasion to see good research and effective teaching activities taking place together. This was for me a most encouraging experience. 